\title{
A CASE FOR INCREASED CAUTION IN END OF LIFE DECISIONS FOR DISORDERS OF CONSCIOUSNESS
}

Jakob Hohwy, Monash University

David C. Reutens, The University of Queensland

Disorders of consciousness include coma, the vegetative state and the minimally conscious state. Such patients are often regarded as unconscious. This has consequences for end of life decisions for these patients: it is much easier to justify withdrawing life support for unconscious than conscious patients. Recent brain imaging research has however suggested that some patients may in fact be conscious.

We argue that these new findings should lead us to be more cautious with regard to end of life decisions for this patient group. Additionally, we argue that if their lives are to be ended, then increased caution should be exercised to avoid undue suffering.

As a consequence, the already difficult ethical and clinical questions surrounding these patients are made even more difficult with regard to making and acting on end of life decisions, as well as with regard to quality of life prognoses. The best we can hope for is that research both on disorders of consciousness and on the neural correlates of consciousness will progress more and make these kinds of questions easier to address in the future.

\section{INTRODUCTION}

Disorders of consciousness include coma, the vegetative state (VS) and the minimally conscious state (MCS). Commonly, patients in coma and VS are regarded as unconscious. This has consequences for end of life decisions for these patients: it is much easier to justify withdrawing life support for unconscious than conscious patients.

Recent brain imaging research has however suggested that some VS patients may in fact be conscious (Owen et al. 2006). In particular, one patient exhibited brain activity comparable to normal, conscious subjects when she was asked to imagine playing tennis and navigating through her house. So far only few such cases have been reported but more studies are under way in this very active research area.

The question is: do findings such as these change the situation in regard to end of life decisions for this patient group? Here we present a case that it does. We argue that the studies should lead us to be more cautious than we presently are in ending these pa- 
tients' lives. Moreover, we argue that if their lives are to be ended, then the new findings should lead to increased caution with respect to avoiding undue suffering when withdrawing life-support.

The consequence of a call for more caution is, in the first instance, that the already difficult ethical and clinical questions surrounding patients with disorders of consciousness are made even more difficult both in terms of end of life decisions and quality of life prognoses. The best we can hope for is that research both on disorders of consciousness and on the neural correlates of consciousness in general will progress more and make these kinds of questions easier to address in the future.

\section{HOW TO DEAL WITH THE NEW EVIDENCE}

The study that has received most attention is the mentioned one by Adrian Owen and colleagues at Cambridge (Owen et al. 2006). A young woman with a traumatic head injury had been in a vegetative state for a long period of time. In the critical part of this study, a fMRI scan was performed while she was asked to follow two different kinds of instructions: to imagine playing tennis or to imagine navigating through the rooms of her home. The tasks were chosen because they have very robust fMRI signatures in the healthy brain, for example, tennis imagery is known to activate the supplementary motor area (SMA). A number of healthy volunteers were also scanned under similar conditions. As would be expected, given earlier studies, the volunteers' SMAs showed activity when they were asked to imagine playing tennis. The crucial finding was that the VS patient's SMA showed comparable levels of sustained activity when she was asked to imagine tennis too. This seems to be evidence that she too is imagining tennis, a task that we normally associate with being conscious. It is also evidence that she is able to follow instructions, which again is something we typically associate with being conscious.

How does the occurrence of such evidence for VS-consciousness change the situation in regard to end of life decisions for these patients and other patient groups with severe, long-term disorders of consciousness (we are not here concerned with more transient loss of consciousness such as epileptic seizures)? Before this evidence became available, our best opinion was that VS patients were unconscious. This made it easier to justify ending their lives since it is much easier to justify ending the life of a creature without consciousness than it is to justify ending the life of a creature that is conscious. Notice that the point here is not that it is ever easy to justify ending the life of an unconscious creature, only that it seems uncontroversial that it is easier than in the case of a conscious creature. 
The new evidence of VS-consciousness is not conclusive evidence of VS-consciousness but it does increase the probability that VS patients are conscious. And it should make us expect more evidence in support of VS-consciousness (we are told that much more such evidence is in fact in press).

So, after getting the evidence for VS-consciousness we should believe that it is more probable that the patients or some of them are conscious, and this accordingly makes it less easy to justify ending their lives. The new evidence must therefore be ethically relevant, unless one can completely refute the findings of the studies. The point here is not that the new evidence must radically change the ethical state of play in respect of VS patients, only that it must change it to some degree dependent on the strength of the evidence.

Given that it is now less easy to justify ending these patients' lives, it would be prudent to be more cautious, consummate with the strength of the evidence, when making end of life decisions for them.

\section{HOW CAUTIOUS SHOULD WE BE?}

We do not believe the new evidence can be plausibly completely refuted as being at least some degree of evidence of some sort or degree of consciousness in VS patients. The only thing to discuss is therefore how strong the evidence is, and what sort of consciousness it is evidence for. The crucial point to notice, before entering this important discussion, is that the very fact that we are having this discussion is an indication that it has become harder to justify ending VS patients' lives and thus that the ethical state of play has changed in favour of caution. What the discussion is about is accordingly how much more cautious we should be.

\subsection{HOW STRONG IS THE EVIDENCE?}

The evidence is clearly not that strong yet since the crucial case in Owen's study is derived from just one patient. More patients that display this kind of brain activity are needed before generalisations can be made with confidence. But the existing study is well-designed and is currently being replicated around the world.

We do not believe it would be convincing to use the limited strength of the evidence to claim there are zero ethical implications from these studies. Perhaps if numerous replications fail this case can begin to be made but that is really dependent on what future studies show.

One objection that has been raised against the Owen study is that it is unable to control for the possibility that the brain activity observed while the patient is asked to 
image playing tennis is automatic and thus not conscious (Naccache 2006; Levy 2008). There is much to discuss in this respect (see, e.g., Owen 2008; Hohwy 2009). It is true that the Owen study doesn't conclusively rule out the possibility that the brain activity is merely automatic. But we do not think this challenges our claim here that more caution is now required when dealing with VS patients. Even if the evidence for VS-consciousness isn't conclusive, it still considerably raises the probability of VS-consciousness and that is enough to make it ethically relevant. This is so particularly when we consider how much is at stake in these decisions: if there is even a small probability that a VS patient is really somewhat like a conscious but totally locked-in patient we must surely be cautious.

For what it is worth, we do not think we have particularly strong reasons to believe the activity is in fact automatic. We also suspect that the main reason the suspicion of automaticity can be cogently raised is that the standard ways of ruling automaticity out cannot be brought to bear on VS patients. Standardly, we simply ask people if they are aware of something but that is precisely the thing we cannot do in VS. But of course, if verbal report is the only thing that could finally convince us that some brain activity was not automatic, then we just beg the question against the VS patients whom by definition cannot give verbal report. In this respect, it will be especially interesting to see the results of current efforts by labs around the world to set up imaged brain activity as a communication system with VS patients. (Roughly, imagine tennis for "yes", imagine navigating your house for "no", then use those imaginings to answer questions. At a conference in June 2009 , the leading neuroscience labs in this field reported that about $10 \%$ of tested patients can communicate in this way. The next couple of years will see an increased number of published studies in this area).

Interestingly, it has not been possible to replicate these findings in minimally conscious (MCS) patients. So it is hard to generalise to that patient group even though they intuitively should be at a higher level of consciousness than VS patients. On the other hand, a different experimental paradigm has suggested a degree of voluntary, endogenous attention in MCS. Many of these patients show a characteristic EEG response when asked to count occurrences of their own name or another's name when listening to a list of names (Demertzi et al. 2008). This serves also as a reminder that the tennis imagery paradigm is not the only one that could be relevant for showing consciousness in disorders of consciousness. 


\subsection{WHAT IS IT EVIDENCE OF?}

It is thus not plausible to deny that the studies increase the probability of VS-consciousness. In order to sideline the ethical consequences of the new evidence of VS-consciousness one would instead have to make a credible case that in so far as the evidence is evidence of consciousness it is evidence of an ethically irrelevant type of consciousness, or at least a type of consciousness that is irrelevant for end of life decisions. This is a doubly difficult task. It requires us to specify which type of consciousness is relevant for end of life decisions, and to determine whether the intact brain areas in VS-patients can sustain that type of consciousness. It is, as we shall suggest below, extremely difficult to make an uncontroversial verdict on these questions, so no kind of verdict on the questions is likely to refute our claim that the new evidence has made it harder to justify ending the lives of VS-patients.

There are various candidates for being the kind of consciousness that is relevant to ethical decisions, in particular end of life decisions:

Phenomenal consciousness. This is the mere presence of occurrent conscious experience, for example of pain, thirst, the smell of a rose, the sound of one's name or indeed the mental image of playing a tennis forehand. In philosophy this is captured by the phrase that "there is something it is like" for a given creature (Nagel 1974).

Access consciousness. This is the availability of phenomenally conscious content to various cognitive consumer systems in the brain such as memory, planning, and verbal report (Block 1995; Block 2008).

It is possible to claim (i) that the new evidence at most is evidence of phenomenal consciousness, not of access consciousness, together with the (not uncontroversial) (ii) that only access consciousness is relevant for end of life decisions (see, e.g., Horne, and Levy this issue). Regardless of the moral underpinnings of claim (ii), this package of claims is hostage to the fate of the empirical hypothesis that phenomenal and access consciousness can dissociate, and this is an extremely controversial issue (see, e.g., the many peer responses to Block 1995; Block 2008). Should it turn out that they don't dissociate, then the package of claims reduces to the claim that no consciousness whatsoever is made probable in studies likes Owen's, and we have argued that this is not a very convincing claim. Moreover, though there is evidence that a degree of neural interconnectivity is necessary for access consciousness (Baars 1997; Tononi 2005; Tononi \& Koch 2008) not much is known about how little such interconnectivity is needed for some degree of access, or for a degree of access sufficient to warrant end-of-life relevance (presumably complete access to all systems is not required). Similarly, even on the most 
recent and most sophisticated approach to investigating the neural correlates of the contents of phenomenal consciousness there is ample scope for interpretation of the extent to which consciousness is really being studied (Haynes 2009; Hohwy 2009).

State consciousness (sometimes called creature consciousness). This is one's overall state of consciousness, quite apart from which particular conscious experiences happen to inhabit consciousness at any given time (Rosenthal 1986; Bayne 2007). It is the type of consciousness one gains on waking after dreamless sleep, and that one loses when one is anesthetized. VS patients have a sleep-wake cycle and the crucial question is of course whether they are in a conscious state at all while in the wake part of that cycle. There is evidence that when patients recover from VS, and can verbally report their conscious states and score high on behavioural ratings such as the Glasgow Coma scale, they regain a characteristic, widely distributed pattern of neural connectivity going back and forth between the thalamus deep in the brain and the cortex (Laureys et al. 2000). But the argument cannot be made that therefore the patients are not in an overall conscious state while vegetative. It may be that the thalamocortical activity pattern is primarily something that enables behaviourally reporting conscious states rather than enabling the conscious state itself. Finally, next to nothing is known about how phenomenal, access and state consciousness relate to each other, or indeed of their neural substrates and how damage to the brain may interfere with these substrates.

Self-consciousness, including the ability to reflexively have plans for the future. This is the ability to engage in self-related reasoning, conceiving of a future for one-self, making plans for that future based on one's memory of the past, and so on. This notion goes further than the other types of consciousness in as much as it requires that those types are related in some sense to the having of a planning self. The argument can be made that only this type of consciousness is relevant for end of life decisions (for discussion, see Levy, Horne, this issue). In philosophy of mind and cognitive neuroscience there is very little agreement about what exactly it is to have self-consciousness or indeed how to study the self experimentally (Gallagher 2000). So even if it was plausible to say that only this kind of consciousness is relevant for end of life decisions, it would be very hard to make a case confidently that a VS patient has or has not sufficient intact brain structures to support it.

The most relevant set of findings with respect to self-related reasoning such as envisioning a future for oneself probably comes from research on the so-called default mode network of the brain. This is a network of the brain (including the medial prefrontal cortex, posterior cingulate cortex and parts of the parietal cortex), which shows relative activity increase during self-related tasks and less activity when subjects are engaged in 
attention demanding tasks, and vice versa (Buckner et al. 2008; Schacter et al. 2008). Whereas there is good reason to believe that this area is relevant for having self-related thoughts, less is known of its implication in sustaining self-consciousness, and even less in regard to a subject's conscious interest in a particular future. No study has to our knowledge looked at activity in the default mode network when VS patients are asked to perform a self-related vs attention demanding task. A recent review did find that the default mode network in disorders of consciousness shows fairly normal activity during rest, ie when no task is performed (Boly et al. 2008). This suggests at least that its activity during rest can be preserved in disorders of consciousness and leaves it not implausible that it could be engaged in self-related tasks too, even if sensory processing is heavily compromised. This is something, again, for future research to reveal.

Given the still sparse empirical evidence concerning all of these types of consciousness and their potential connectivity, it is very difficult to arrive at any reasonably confident conclusion about the exact type of consciousness that Owen's study may be evidence of. This means that it will be very hard indeed to justifiably rule out that it is a type of consciousness that is relevant to end of life decisions.

\subsection{IS PHENOMENAL CONSCIOUSNESS IRRELEVANT?}

So far we have not engaged directly the ethical issues about which type of consciousness is relevant for end of life decisions - we have just focused on the empirical sensitivity of such claims. Now we briefly turn to the ethical issues.

Sometime in the (rather distant) future consciousness science might be able to identify robust neural correlates of these different types of consciousness. It might then be that we find that in so far as there is consciousness in VS-patients, it is predominantly consistent with activity in the neural correlate of phenomenal consciousness. Would this imagined situation (for which there is no evidence now) be sufficient to make the verdict that their consciousness is not relevant for end of life decisions? The argument would be that with little in the way of access consciousness to cognitive consumer systems and self-related planning for the future there is little reason to treat them as having a right to life (for discussion, see Levy this issue).

The first thing is to note that evidence about the neural correlates of consciousness is procured mainly from healthy subjects and it can be difficult to extrapolate to cases where there is extensive brain damage (Chalmers 2000). So consciousness science would have to have evolved to a very advanced stage indeed where even the neural correlates of abnormal consciousness are revealed. 
The ethical claim can also be scrutinized. According to the kind of view in question, mere phenomenality is deemed insufficient for a right to life (though deemed relevant for being a moral patient for whom pain, for example, should be avoided). Instead selfrelated processing and conscious access is what is needed. We believe it is very difficult to make a confident call on how much self-related processing or conscious access one needs to qualify as having a right to life.

Consider one of the most famous subjects in the history of cognitive neuroscience, HM. To treat severe epilepsy HM had large parts of his medial temporal lobe removed. The result was severe anterograde amnesia such that he was unable to form any long term episodic memories of events occurring after the surgery (Scoville \& Milner 1957). He would thus retain most memory from before the surgery but live in the short moving window of a couple of minutes sustained by his working memory. HM would therefore be rich in phenomenal consciousness and he would presumably be able to form long term, self-conscious plans for the future as long as doing so wouldn't outstrip working memory. It seems plausible to surmise that he would nevertheless be heavily impaired in terms of truly being able to engage in long-term planning of his future given he would be unable to remember what he had decided to do. There would not be much to differentiate such long-term planning from idle confabulation. And yet, very obviously, it would be ludicrous to deny HM the right to life just because he cannot engage in meaningful long-term planning. We suspect that our reaction to HM's case is in part driven by acknowledging that he was indeed having a rich phenomenally conscious life, albeit in small snippets of time. So we suspect that it is a mistake to relegate merely phenomenal consciousness to being irrelevant for right to life issues. To elaborate, imagine that VS patients are somewhat like HM just with a narrower moving window of working memory. They may have phenomenal experience and be able to plan for the future but only very briefly. It doesn't seem attractive to say that the difference in how narrow the moving window is is what makes the difference to these subjects' right to life.

\section{IF THE LIFE OF A SUBJECT WITH A DISORDER OF CONSCIOUSNESS MUST BE ENDED, THEN INCREASED CAUTION MUST BE EXERCISED TO END IT WITHOUT UNDUE SUFFERING}

In this final section we change topics somewhat. So far we have been arguing that the new imaging evidence of VS-consciousness obligates us to be more cautious when making end of life decisions for this group. Now we wish to consider briefly how to proceed if a decision has been made to in fact end such a patient's life. Normally, and for legal 
reasons, this is done by withdrawing life support (such as feeding tubes) and "letting nature take its course". The question is whether there is evidence that suggests we should re-think how end of life decisions are executed.

If a patient is wholly unconscious, then whether her life ends swiftly or not is not so important. Ethical considerations in this case mainly concern relatives. It can be painful to be with a loved one who is slowly dying, and it can be brutal to see someone swiftly euthanized.

But if the VS patient is in fact conscious the situation is different. Clearly, someone conscious should have a painless and dignified death. So again we see that it matters greatly what we say about studies that raise the probability that such patients are conscious. It might also seem that it matters here what type of consciousness is preserved in these patients. But here it seems uncontroversial that mere phenomenal consciousness matters greatly. If a patient is able to feel pain and thirst, then we are morally obligated to choose a method of death that minimises the probability that they have those emotions and sensations. Evidence is available that many patients with disordered consciousness and other disorders (such as hydranencephaly, see Merker 2007) may in fact have phenomenal experience of raw emotions of pain and thirst, irrespective of whether they have cognitive access to these or can slot them into self-conscious plans for the future (Panksepp et al. 2007). Panksepp makes the obvious recommendation that follows from this, namely that if their lives must be ended, then they must be ended such as to minimise these extremely unpleasant raw emotions. Analgesics may be given but do not necessarily deal with other raw emotions such as thirst and palliative sedation may be required to blunt these sensations.

Panksepp equates this requirement to minimise unpleasant raw emotions with introducing a more proactive and swift method than withdrawal of life-support. We do not think time itself is of the essence as it is possible to kill swiftly and painfully (possible trauma in the survivors must also be considered). But the core point remains that from an ethical perspective the normal withdrawal method, in the absence of other palliative measures, is put under pressure. Now that the probability is significantly increased that VS patients have a relevant degree of consciousness, the emphasis is placed on managing end of life situations proactively to minimise suffering.

While the use of palliative approaches fall within current legal guidelines, Panksepp and colleagues have raised the possibility of more interventionist approaches to executing end of life decisions which comprise a challenge to existing laws. 


\section{CONCLUSION}

There is new evidence that suggests that some patients in the vegetative state may have some form of consciousness. The question is how if at all this changes the ethical issues surrounding the patients' moral status, in particular in respect of end of life decisions. We have argued that it is highly implausible to hold that the new evidence leaves all the ethical matters unchanged. Even though the new evidence is not conclusive it is already strong enough to be un-ignorable. Similarly, the nascent state of consciousness science does not allow a conclusion that the neural damage typically seen in VS undermines ethically relevant consciousness.

Given that the probability of VS-consciousness has risen with this new evidence, we should be more cautious when confronted with the difficult ethical questions of whether or not to withdraw life support. In addition, if a decision to withdraw life support is made, active interventions are required to ensure that suffering is alleviated in the potentially conscious patient.

What does "more cautious" mean? Obviously, clinicians are already extremely cautious to get the diagnosis of vegetative state right. Much care is now taken, for instance, to rule out the locked-in state. To be more cautious means, basically, that these clinical decisions are now made even more difficult: clinicians must now take into consideration that the patient may be in some kind of locked-in state but without any kind of outward behavioural sign, like eye movement. Moreover, the exact type of locked-in consciousness that may be present is still uncertain, meaning that even if some inward behavioural sign could be found (e.g., the mentioned imaging communication system) it may still be hard to interpret what it being communicated.

A crucial aspect of end of life decisions concerns what kind of life the patient is likely to have. A likely poor quality of life may heavily influence the decision in favour of withdrawing life support. Normal intuitions vary a lot here. We wonder whether being conscious but unable to speak and control and interact with one's environment is necessarily a wretched life. It is true that one is less in control of one's quality of life and so it may be more easily made into a wretched life through neglect, for example. But given the right circumstances it may not be a bad way to be. Perhaps it would feel like being half asleep not wanting to do anything, which is no bad thing in a cosy bed on a cold Sunday morning. But we really do not know, it may also be a life wrought with fear and pain, in which case indecision, delays and lack of resources on the part of the carers may have significant negative effects. Another issue is the quality of life that the patient is likely to have if, further down the track, he or she recovers some degree of consciousness. 
This aspect of end of life issues is also made more complicated and again more caution is urged. The reason is that presumably the prognosis for an individual patient depends to a significant degree on the cognitive and conscious abilities that are preserved while still in the vegetative state. If they are reasonably conscious in VS, then their prognosis will be better. Now that there is evidence that some but not all patients are conscious it seems prudent to factor that in, difficult as it may be, when considering their prognosis.

End of life decisions are made and must continue to be made for patients with disorders of consciousness. The call for increased caution will not be easy to honour in the clinical setting. The best we can hope for is therefore that much more research is done that will allow more informed decisions about the consciousness of these patients. This includes not only research on the patients themselves, in the style of Owen, Laureys, Naccache and others, but also an increased effort in basic consciousness science to find the neural correlates of different aspects of consciousness in the healthy population.

\section{REFERENCES}

Baars, B.J. 1997. In the Theater of Consciousness: The Workspace of the Mind. Oxford, Oxford University Press.

Bayne, T. 2007. 'Conscious states and conscious creatures: Explanation in the scientific study of consciousness'. Philosophical Perspectives 21 (1): 1-22.

Block, N. 1995. 'On a confusion about a function of consciousness'. Behavioral and Brain Sciences 18: 227-287.

Block, N. 2008. 'Consciousness, accessibility, and the mesh between psychology and neuroscience'. Behavioral and Brain Sciences 30 (5-6): 481-499.

Boly, M., Phillips, C. et al. 2008. 'Intrinsic brain activity in altered states of consciousness'. Annals of the New York Academy of Sciences 1129 (Molecular and Biophysical Mechanisms of Arousal, Alertness, and Attention): 119-129.

Buckner, R.L., Andrews-Hanna, J.R. et al. 2008. 'The brain's default network: Anatomy, function, and relevance to disease'. Annals of the New York Academy of Sciences 1124 (1): 1-38.

Chalmers, D. 2000. 'What is a neural correlate of consciousness?' Neural Correlates of Consciousness: Empirical and Conceptual Issues. T. Metzinger. Cambridge, Mass., MIT Press.

Demertzi, A., Vanhaudenhuyse, A. et al. (2008). 'Is there anybody in there? Detecting awareness in disorders of consciousness'. Expert Review of Neurotherapeutics 8 (11): 1719-1730.

Gallagher, S. 2000. 'Philosophical conceptions of the self: implications for cognitive science'. Trends in Cognitive Sciences 4 (1): 14.

Haynes, J.D. 2009. 'Decoding visual consciousness from human brain signals'. Trends in Cognitive Sciences 13 (5): 194-202.

Hohwy, J. 2009. 'The neural correlates of consciousness: New experimental approaches needed?' Consciousness and Cognition 18 (2): 428-438. 
Laureys, S., Faymonville, M.E. et al. 2000. 'Restoration of thalamocortical connectivity after recovery from persistent vegetative state'. Lancet 355: 1790-1791.

Levy, N. 2008. 'Going beyond the evidence'. The American Journal of Bioethics 8 (9): 19-21.

Merker, B. 2007. 'Consciousness without a cerebral cortex: A challenge for neuroscience and medicine'. Behavioral and Brain Sciences 30 (1): 63-81.

Naccache, L. 2006. 'Is she conscious?' Science 313: 1395-1396.

Nagel, T. 1974. 'What is it like to be a bat?' Philosophical Review 83: 435-450.

Owen, A.M. 2008. 'Disorders of consciousness'. Annals of the New York Academy of Sciences 1124 (1): 225-238.

Owen, A.M., Coleman, M.R. et al. 2006. 'Detecting awareness in the vegetative state'. Science 313 (5792): 1402.

Panksepp, J., Fuchs, T. et al. 2007. 'Does any aspect of mind survive brain damage that typically leads to a persistent vegetative state? Ethical considerations'. Philosophy, Ethics, and Humanities in Medicine 2 (1): 32.

Rosenthal, D.M. 1986. 'Two concepts of consciousness'. Philosophical Studies 94: 329-359.

Schacter, D.L., Addis, D.R. et al. 2008. 'Episodic simulation of future events: Concepts, data, and applications'. Annals of the New York Academy of Sciences 1124 (1): 39-60.

Scoville, W.B. and Milner, B. 1957. 'Loss of recent memory after bilateral hippocampal lesions'. Journal of Neurology, Neurosurgery and Psychiatry 20: 11-21.

Tononi, G. 2005. 'Consciousness, information integration, and the brain'. Progress in Brain Research. S. Laureys, Elsevier. Volume 150: 109-126.

Tononi, G. and Koch, C. 2008. 'The neural correlates of consciousness: An update'. Annals of the New York Academy of Sciences 1124: 239-261.

Cite this article as: Hohwy, Jakob; Reutens, David C. 'A case for increased caution in end of life decisions for disorders of consciousness'. Monash Bioethics Review 28 (2): pp. 14.1 to 14.12. DOI: 10.2104/mber0914. 